\title{
The Design of Resource Management Mechanism with Hybrid Access in a Macro-Femto System
}

\author{
Binglai Niu and Vincent W.S. Wong \\ Department of Electrical and Computer Engineering \\ University of British Columbia, Vancouver, Canada \\ e-mail: \{bniu, vincentw\}@ece.ubc.ca
}

\begin{abstract}
In this paper, we study uplink resource management in a two-tier macro-femto heterogeneous system with hybrid access control. We consider a system where a macro base station (MBS) and a femto base station (FBS) together serve a number of mobile users. In this system, base stations and users make decisions in various resource management processes with different optimization objectives. Such decision making processes are usually correlated and an efficient mechanism is needed to coordinate the decision makers. We propose a fourstage resource management mechanism where access control, resource allocation (in both frequency and time domain) and power control are performed sequentially at the base stations and users, respectively. We formulate a resource management problem and find the optimal decisions for base stations and users. We show that this mechanism provides incentive for the FBS to operate in hybrid access mode. Simulation results show that the proposed resource management mechanism achieves a better performance than the mechanism with closed access.
\end{abstract}

\section{INTRODUCTION}

Recently, with the increasing demand of mobile data services, there is an emerging trend of deploying low cost and low power base stations overlay the existing cellular infrastructure to extend the coverage area and increase the system capacity. In particular, in urban residential area, installing femto base stations (FBSs) in houses and apartments has been considered as an efficient approach to improve the indoor signal quality [1]. FBSs are installed by users and can serve a small number of subscribers at the operator's licensed spectrum. By deploying FBSs, home users' devices can experience better indoor signal quality and longer battery life due to the close proximity [2]. Despite the promise, the deployment of FBSs also introduces technical challenges, such as interference management between the macrocell and femtocells, and coordination among resource management processes [3]. Extensive research has been performed on interference management in heterogeneous networks, and several approaches have been proposed to improve the system performance, such as frequency planning, orthogonal resource allocation, and power control [4].

Recent attention on two-tier heterogeneous systems has been drawn to designing efficient resource management mechanisms (i.e., channel allocation, power control) with access control at the FBSs [5]-[12]. Since FBSs are deployed by users, typically they are configured to operate in closed access mode and only serve authorized subscribers, referred as femtocell users (FUs). However, it has been shown that allowing non-subscribers, i.e., macrocell users (MUs), to access FBSs can improve the overall system performance when the MUs experience poor signal quality from the macro base station (MBS) [5]. As a result, hybrid access mode has been proposed to deal with this issue. In hybrid access mode, the FBS may grant access to MUs with limited resources while serving its subscribed FUs with higher priority. This access mode aims to seek a balance between improving the overall system performance and preserving the quality of service (QoS) at the FUs, and has become a popular choice in designing novel resource management mechanisms [6].

The emergence of hybrid access mode brings new challenges to designing resource management mechanisms for heterogeneous networks. First, since an FBS is owned and installed by FUs, it may not offer free services for MUs. Therefore, incentives should be provided to motivate the FBS to operate in hybrid access mode. In the literature, spectrum leasing schemes have been proposed to encourage hybrid access, where the MBS leases the spectrum to the FBSs, and the FBSs further lease their spectrum to nearby MUs at certain prices [7], [8]. Although incentives can be provided, such schemes are developed based on specific business models, which may not be available in existing systems. In practice, an MU may pay access fee to the service provider instead of making separate payments to FBSs. Therefore, it is interesting to study how to encourage the FBSs to adopt hybrid access without modifying the business model, which motivates the research in this paper.

In addition to providing incentives, an efficient mechanism is needed to coordinate the access control and other resource management processes. The access control decisions at FBSs may affect the channel allocation decisions at the MBS and power control decisions at the users. For instance, when allocating channels, the MBS may need the access control decisions (or user association profile) to optimize the overall system performance. An MU may also need to know which base station it is associated with to determine its transmission power. Therefore, optimization of access control, resource allocation and power control should be jointly considered when designing resource management mechanisms. Most of the existing works adopt heuristic access control mechanism such as topology based mechanism [10] or interference based mechanism [11], while the optimization of access control decisions together with other resource management processes has not been well explored. 


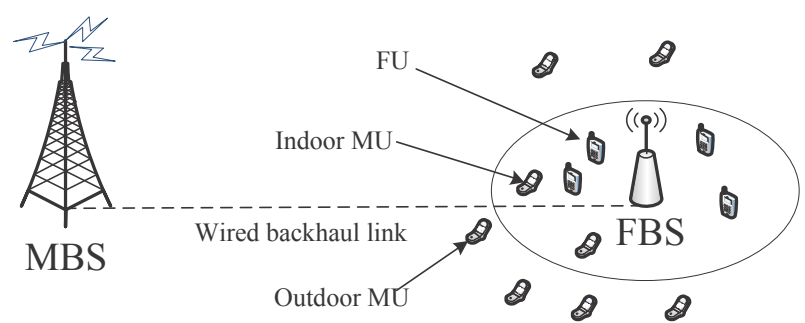

Fig. 1. Two-tier macro-femto system model. The MBS and the FBS together serve a number of users that are close to the FBS

In this paper, we propose a resource management mechanism to coordinate the access control, resource allocation and power control processes for a macro-femto heterogeneous system. We model the resource management process as a fourstage decision making process and find the optimal decisions for base stations and users. Our work differs from others in three aspects. First, instead of using pricing based incentive mechanisms, we propose an adaptive channel allocation scheme to encourage hybrid access at the FBS. Second, we consider base stations and users are individual decision makers with different objectives, and design a mechanism to coordinate their decision making processes. Moreover, we jointly optimize the access control with other resource management processes at the base stations and users, respectively.

The rest of this paper is organized as follows. In Section II, we describe the system model and formulate the resource management problem. In Section III, we analyze the problem and find the optimal solution for each resource management stage. Simulation results are presented in Section IV, and conclusions are drawn in Section V.

\section{System Model ANd PRoblem Formulation}

We study the uplink of a two-tier macro-femto wireless system, where an MBS and an FBS together serve a number of users as shown in Fig. 1. The FBS connects to the MBS via a wired backhaul link. We assume the capacity of the backhaul link is large enough to handle the data transmission for a small group of users. We denote the set of base stations as $\mathcal{I}=\{M, F\}$, where $M$ represents the MBS and $F$ represents the FBS. There is a set of FUs served by the FBS, which is denoted as $\mathcal{F}$. The other users in the system are MUs that are close to the FBS, and we denote the set of the MUs as $\mathcal{M}$. This model represents the cellular network in residential area where an FBS is installed by home users to improve the service quality. Note that although we present the proposed resource management mechanism under this simple system model, this mechanism can be extended to systems with multiple FBSs with proper adjustments.

We consider a time slotted system, where the time frame is divided into slots of equal length. The total number of available channels for the system is $N$, and each channel has a bandwidth of $B$. We assume a frequency flat block fading wireless channel model, where for any of the $N$ available channels, the channel fading between a user and its base station remains constant during a time slot and is independent and identically distributed (i.i.d.) in different time slots with zero mean and unit variance complex Gaussian distribution $(\mathcal{C}(0,1))$. The MBS is responsible for allocating the available channels to the FBS and itself. For the FBS, based on the aforementioned i.i.d. channel model, we only consider determining the number of channels that should be allocated to it. Since the MUs are close to the FBS, to avoid co-channel interference, we assume the MBS and the FBS are allocated different channels. Users associated with the same base station share the available resources (channels and time slots) using time-division multiple access and at most one user can access a particular channel during a time slot.

The FBS is owned by FUs and can choose to operate in the hybrid access mode, where the FBS may allow some MUs to access it but reserves a proportion of the available resources for its own FUs. We define the set of MUs associated with the FBS as $\mathcal{S}_{F}^{m}$ when hybrid access mode is used, where $\mathcal{S}_{F}^{m} \subseteq$ $\mathcal{M}$. Thus, the set of all users associated with the FBS (when hybrid access mode is used) is denoted as $\mathcal{S}_{F}=\mathcal{S}_{F}^{m} \cup \mathcal{F}$. We also define the set of users associated with the MBS as $\mathcal{S}_{M}$. Users' devices have the capability to sense the signal quality from nearby base stations (based on pilot signals) and an MU may send a request to access the FBS that provides the best signal quality. Users can also choose transmission power from the set $\mathcal{P}=\left\{0, P_{l 1}, \ldots, P_{l L}, P_{\max }\right\}$ to optimize their utilities. We consider each user requests a service (e.g., video chatting) that requires a minimum average data rate (over a transmission period) to guarantee its QoS. We assume the total amount of resources in the system is sufficient to satisfy the QoS requirements for all the users.

We consider that there is a resource management process to setup system parameters prior to users' data transmission, which includes access control, resource allocation, and power control at the base stations and users. Such process is performed periodically, i.e., every $T$ time slots. After the resource management process, users start their data transmission.

\section{A. Utility Function}

We define utility functions to characterize the objectives at the users and base stations. In practice, a wireless user prefers receiving high QoS with low energy consumption. Therefore, we define the utility function for user $i \in \mathcal{S}_{j}$ who is associated with base station $j \in \mathcal{I}$ as

$$
u_{i}=f_{i}\left(\bar{R}_{i j}\right)-c_{i}\left(w_{i j}, \bar{P}_{i}\right),
$$

where $w_{i j} \in\left\{\frac{1}{T}, \frac{2}{T}, \ldots, 1\right\}$ is the proportion of time that user $i \in \mathcal{S}_{j}$ is granted access to base station $j \in \mathcal{I}$ over one channel during the transmission period. $f_{i}(\cdot)$ characterizes the user's satisfaction with respect to the average data rate over one transmission period $\left(\bar{R}_{i j}\right)$, and $c_{i}(\cdot)$ is a function of the average transmission power $\left(\bar{P}_{i}\right)$ and $w_{i j}$, which characterizes the energy consumption of user $i$ 's device.

According to [13], a user's satisfaction is an increasing function of the data rate, which has a decreasing marginal improvement as the data rate increases. Such property can be modeled using concave functions. In this work, to characterize 
users' satisfaction with QoS requirement, we consider a satisfaction function $f_{i}(\cdot)$ as follows. For user $i \in \mathcal{S}_{j}$ associated with base station $j \in \mathcal{I}$, we have

$f_{i}\left(\bar{R}_{i j}\right)= \begin{cases}\alpha \ln \left(1+\left(\bar{R}_{i j}-R_{i}^{\mathrm{min}}\right)\right)+\gamma, & \text { if } \bar{R}_{i j} \geq R_{i}^{\mathrm{min}}, \\ 0, & \text { otherwise, }\end{cases}$

where $R_{i}^{\min }$ is the minimum average data rate required to guarantee the basic QoS for user $i, \alpha$ and $\gamma$ are positive constant parameters. From (2), it can be seen that user $i$ gains nothing when its data rate is less than the basic QoS requirement. When the data rate is greater than the QoS threshold, the user's satisfaction becomes a concave function of the data rate.

We assume each user chooses a fixed transmission power during the transmission period ( $T$ time slots), that is $\bar{P}_{i}=P_{i}$ for $i \in \mathcal{S}_{j}, j \in \mathcal{I}$. We propose to use

$$
c_{i}\left(w_{i j}, P_{i}\right)=\beta w_{i j} P_{i},
$$

where $\beta$ is a positive constant parameter. Note that the average data rate of a user depends on the channel conditions within the future $T$ time slots, which is unknown prior to the resource management process. Based on the channel capacity in [14], we approximate the average data rate by using the expected signal-to-noise ratio (SNR) during the calculation. Specifically, for user $i$ who communicates with base station $j$, the approximate data rate is

$$
\widetilde{R}_{i j}=w_{i j} B \log _{2}\left(1+\overline{\mathrm{SNR}}_{i j}\right)=w_{i j} B \log _{2}\left(1+\frac{g_{i j} P_{i}}{\sigma^{2}}\right),
$$

where $\overline{\mathrm{SNR}}_{i j}=\mathbb{E}\left[\frac{g_{i j}\left|h_{i j}\right|^{2} P_{i}}{\sigma^{2}}\right]=\frac{g_{i j} P_{i}}{\sigma^{2}}, g_{i j}$ is the average channel gain from user $i$ to base station $j, h_{i j}$ is the corresponding small scale fading and $\sigma^{2}$ is the noise power. Here, we use the fact that $\mathbb{E}\left[\left|h_{i j}\right|^{2}\right]=1$ for $h_{i j} \sim \mathcal{C}(0,1)$. In the remaining of the work, we use the approximate data rate to calculate the utility in the resource management process.

We assume each base station's objective is to maximize the total utility of its own users (MUs or FUs), and we denote the corresponding utility for the MBS and FBS as $U_{M}=\sum_{i \in \mathcal{M}} u_{i}$ and $U_{F}=\sum_{i \in \mathcal{F}} u_{i}$, respectively. Note that the proposed configuration mechanism and corresponding analysis in this work can also be applied to other utility functions with proper adjustment.

\section{B. Resource Management Problem}

In the aforementioned macro-femto system, base stations and users make decisions in various resource management processes with different optimization objectives. To coordinate the decision making processes, we propose a four-stage resource management mechanism that follows the procedure as shown in Fig. 2. In Stage I, after receiving the access requests from the MUs, the FBS determines its association profile $\boldsymbol{a}_{F}=\left(a_{i}, i \in \mathcal{M}\right)$, where $a_{i}=1$ indicates that MU $i$ is granted access to the FBS. Then, the FBS sends its access control decision $\left(\boldsymbol{a}_{F}\right)$ to the MBS. In Stage II, the MBS performs channel allocation based on the feedback information

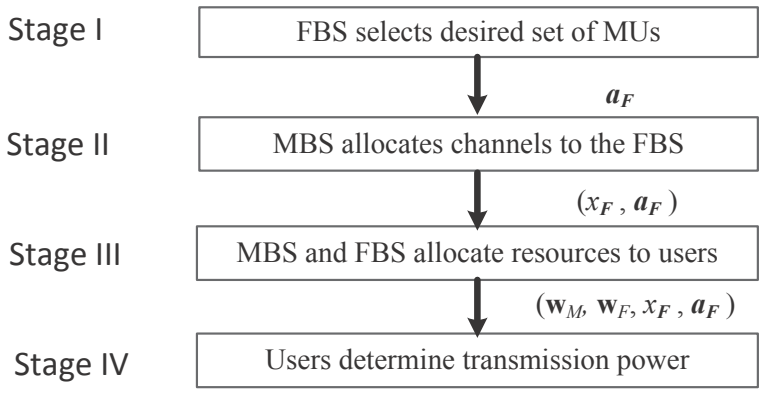

Fig. 2. Four-stage resource management mechanism: Stage $I$ is the access control process, Stages II and III correspond to the resource allocation process and Stage IV is the power control process.

from the FBS, i.e., it determines the number of channels to be allocated to the FBS, denoted as $x_{F}$. To motivate hybrid access at the FBS, we adopt an adaptive channel allocation scheme at the MBS, which will be introduced later in this work. After channel allocation, the MBS and the FBS allocate their available resources (in both frequency and time domains) to their associated users in Stage III, i.e., base station $j \in \mathcal{I}$ determines its resource allocation profile $\mathbf{w}_{j}=\left(w_{i j}, \forall i \in \mathcal{S}_{j}\right)$. Finally, each user $i$ determines its transmission power $P_{i}$ that maximizes its utility based on the available resources $\left(w_{i}\right)$.

We define $\boldsymbol{a}_{F}^{*}, x_{F}^{*}, \mathbf{w}_{j}^{*}(j \in \mathcal{I})$, and $P_{i}^{*}(i \in \mathcal{M} \bigcup \mathcal{F})$ as the optimal decisions in Stages I, II, III and IV, respectively. In this work, our objective is to find the optimal decisions in each stage, respectively.

\section{Analysis of the Resource Management MECHANISM}

In the proposed resource management mechanism, to determine the optimal access control decision $\left(\boldsymbol{a}_{F}^{*}\right)$ in Stage I, the FBS may require the knowledge of the optimal decisions in the following stages for any given association profile $\left(\boldsymbol{a}_{F}\right)$, since the FBS's objective is to maximize the total utility of FUs, which can only be obtained after the last stage. Therefore, we analyze the resource management problem in a bottom-up manner. We first find the optimal power control decision for each user in Stage IV, given the resource allocation and access control decisions from the previous stages. After knowing the optimal decision at the users, we can find the desired resource allocation strategy at the base stations in Stage III, assuming fixed channel allocation and access control decisions. Then, we derive the desired channel allocation strategy at the MBS in Stage II, and finally find the desired access control strategy for Stage I, subsequently.

\section{A. Power Control at the Users}

We first consider the power control process at users. In this process, a user $i \in \mathcal{M} \bigcup \mathcal{F}$ chooses its transmission power $P_{i} \in \mathcal{P}$ to maximizes its own utility $u_{i}\left(w_{i j}, P_{i}\right)$. Intuitively, given the available resources $\left(w_{i j}\right)$ from base station $j$, user $i \in \mathcal{S}_{j}$ 's optimal power is

$$
P_{i}^{*}=\underset{P_{i} \in \mathcal{P}}{\arg \max } u_{i}\left(w_{i j}, P_{i}\right) .
$$




\section{B. Resource Allocation at the Base Stations}

Next, we study the resource allocation problem at the base stations. We first consider resource allocation at the FBS given its access control decision $\left(\boldsymbol{a}_{F}\right)$ and its available number of channels $x_{F}$ obtained from the MBS. Note that we require the FBS's decision guarantee the QoS requirements of all its associated users, and we assume such decision is feasible (i.e., $x_{F}$ is sufficiently large). The optimal resource allocation decision $\mathbf{w}_{F}$ that maximizes the FBS's utility can be found by solving the following problem:

$$
\begin{array}{clr}
\underset{w_{i F}, i \in \mathcal{S}_{F}}{\operatorname{maximize}} & U_{F}=\sum_{i \in \mathcal{F}} u_{i}\left(w_{i F}, P_{i}^{*}\left(w_{i F}\right)\right) \\
\text { subject to } & \sum_{i \in \mathcal{S}_{F}} w_{i F} \leq x_{F}, & \\
& w_{i F} \geq w_{i F}^{\min }, & \forall i \in \mathcal{S}_{F}, \\
& w_{i F} \in\left\{\frac{1}{T}, \frac{2}{T}, \ldots, 1\right\}, \quad \forall i \in \mathcal{S}_{F},
\end{array}
$$

where $P_{i}^{*}\left(w_{i F}\right)$ is the optimal transmission power for user $i$ given $w_{i F}$, which can be obtained from (5), and $w_{i F}^{\min }$ is the minimum amount of resources that user $i$ needs from the FBS to guarantee its QoS. The first constraint implies that the total amount of resources allocated to users should not exceed the total number of channels available at the FBS. The second constraint guarantees the QoS of all users associated with this FBS. The last constraint implies that a user can only obtain an integer number of time slots and cannot access more than one channel simultaneously. Since $P_{i}^{*}\left(w_{i F}\right)$ also depends on $w_{i F}$, from (1) and (5), it can be seen that problem (6) is a nonlinear discrete optimization problem, which is hard to solve in general. In the rest of this section, we design an efficient algorithm to achieve the optimal solution.

Note that the utility of the FBS is the total utility of its associated FUs. Intuitively, to maximize its utility, the FBS should allocate the minimum amount of resources to the MUs while satisfying the QoS constraints, that is $w_{i F}^{*}=w_{i F}^{\min }, \forall i \in$ $\mathcal{S}_{F}^{m}$. Then, the amount of remaining resources for the FUs is $x_{F}-\sum_{i \in \mathcal{S}_{F}^{m}} w_{i F}^{\min }$, which is a constant. Therefore, solving problem (6) is equivalent to solving the following problem:

$$
\begin{array}{ll}
\underset{w_{i F}, i \in \mathcal{F}}{\operatorname{maximize}} & \sum_{i \in \mathcal{F}} u_{i}\left(w_{i F}, P_{i}^{*}\left(w_{i F}\right)\right) \\
\text { subject to } & \sum_{i \in \mathcal{F}} w_{i F} \leq x_{F}-\sum_{i \in \mathcal{S}_{F}^{m}} w_{i F}^{\min }, \\
& w_{i F} \geq w_{i F}^{\min }, \quad \forall i \in \mathcal{F}, \\
& w_{i F} \in\left\{\frac{1}{T}, \frac{2}{T}, \ldots, 1\right\}, \forall i \in \mathcal{F} .
\end{array}
$$

Based on the above transformation, we propose a two-step algorithm to find the optimal solution for (6), as shown in Algorithm 1. We define $\Delta u_{i}\left(w_{i F}\right) \triangleq u_{i}\left(w_{i F}, P_{i}^{*}\left(w_{i F}\right)\right)-$ $u_{i}\left(w_{i F}-\frac{1}{T}, P_{i}^{*}\left(w_{i F}-\frac{1}{T}\right)\right)$ as the changes of utility when one time slot is assigned to user $i \in \mathcal{S}_{F}$. In the first step of the algorithm, we allocate the minimum number of time slots $\left(w_{i F}^{\min } T\right)$ for each user $i \in \mathcal{S}_{F}$ to satisfy its QoS requirement. This step finds the optimal resource allocation decisions for MUs $\left(w_{i F}^{*}=w_{i F}^{\min }, \forall i \in \mathcal{S}_{F}^{m}\right)$. In the second step, we allocate the remaining time slots one by one, where each time slot is allocated to $\mathrm{FU}(i \in \mathcal{F})$ who has the largest utility increment
$\left(\Delta u_{i}\left(w_{i F}\right)\right)$. This step solves the transformed problem (7), as shown in Appendix A. With Algorithm 1, the following theorem can be proved.

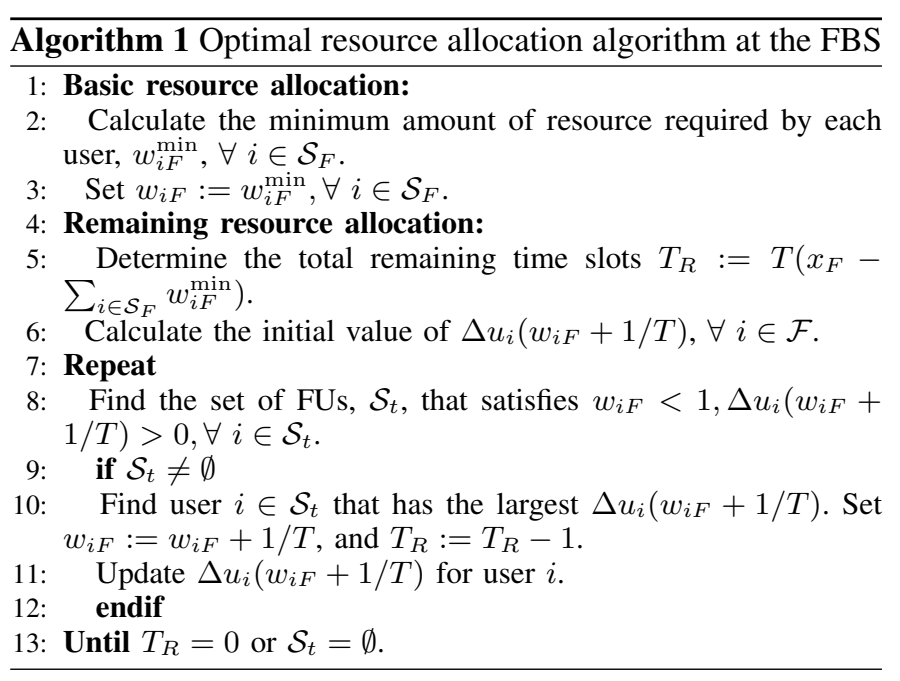

Theorem 1: The resource allocation vector $\mathbf{w}_{F}=$ $\left\{w_{i F}, \forall i \in \mathcal{S}_{F}\right\}$ obtained using Algorithm 1 constitutes the optimal solution to problem (6).

The proof of Theorem 1 is provided in Appendix A. For the MBS, the resource allocation algorithm is similar to that at the FBS, except that the MBS only serves MUs. Therefore, the resource allocation algorithm can be obtained by replacing the terms "FU" by "MU", $w_{i F}\left(w_{i F}^{\min }\right)$ by $w_{i M}\left(w_{i M}^{\min }\right)$, and $\mathcal{F}$ (and $\mathcal{S}_{F}$ ) by $\mathcal{S}_{M}$, respectively, in Algorithm 1. Thus, we have found the desired strategy for the FBS and the MBS in Stage III.

\section{Channel Allocation at the MBS}

In this subsection, we propose a channel allocation scheme at the MBS. First, we consider the FBS operates in closed access mode. In this case, since the MBS's objective is to maximize the total utility of MUs, the MBS prefers to allocate the minimum number of channels to the FBS that guarantees the FUs' QoS, which is defined as $x_{F}^{\min }=\left\lceil\sum_{i \in \mathcal{F}} w_{i F}^{\min }\right\rceil$, where $\lceil\cdot]$ is the ceiling function. This implies that the FBS can obtain at least $x_{F}^{\min }$ channels from the MBS. We denote the resource allocation decision at the MBS (for MUs) in Stage III in this closed access scenario as $\mathbf{w}_{M}=\left(w_{i M}^{c}, i \in \mathcal{M}\right)$, which can be calculated using Algorithm 1 .

Next, we consider the scenario where FBS operates in hybrid access mode and accepts to serve a set of MUs $\left(\mathcal{S}_{F}^{m}\right)$. In this scenario, the MBS should allocate more than $x_{F}^{\min }$ channels to the FBS in order to provide incentives for it to serve the MUs. In this work, we adopt the following channel allocation scheme, where the MBS allocates a number of $x_{F}=x_{F}^{\min }+\left\lfloor\sum_{i \in \mathcal{S}_{F}^{m}} w_{i M}^{c}\right\rfloor$ channels to the FBS, where $\lfloor\cdot\rfloor$ is the floor function. The rational behind this scheme is as follows. If the MUs (in $\mathcal{S}_{F}^{m}$ ) are served by the MBS, they should be allocate an amount of $\sum_{i \in \mathcal{S}_{F}^{m}} w_{i M}^{c}$ resources from the MBS. Since the MUs can obtain better utility from the 
FBS (otherwise they do not send access requests), the MBS offers a number of $\left\lfloor\sum_{i \in \mathcal{S}_{F}^{m}} w_{i M}^{c}\right\rfloor$ additional channels to the FBS as bonus for serving the MUs. The floor function is used to guarantee that the amount of resources allocated to other MUs $\left(\forall i \in \mathcal{M} \backslash \mathcal{S}_{F}^{m}\right.$ ) will not be reduced when the MUs in $\mathcal{S}_{F}^{m}$ change their association from the MBS to the FBS. It can be seen that if the FBS accepts to serve some MUs, it may obtain more channels from the MBS and may preserve more resources to serve its FUs, since the MUs require much less resources from the FBS than that from the MBS to guarantee their QoS. On the other hand, when accessing the FBS, the MUs transmit in fewer time slots compare to that when accessing the MBS, and their utilities can also be improved due to the reduced energy consumption. Base on the proposed channel allocation scheme, we have

$$
x_{F}^{*}=\left\lceil\sum_{i \in \mathcal{F}} w_{i F}^{\min }\right\rceil+\left\lfloor\sum_{i \in \mathcal{S}_{F}^{m}} w_{i M}^{c}\right\rfloor .
$$

\section{Access Control at the FBS}

Finally, we find access control decision at the FBS when multiple access requests are received from the MUs. We define $r_{i}$ as the indicator whether the FBS receives a request from MU $i$, where $r_{i}=1$ indicates the request is received and $r_{i}=0$ otherwise. We denote $\mathcal{A}_{F}=\left\{\boldsymbol{a}_{F} \mid a_{i} \leq r_{i}, \forall i \in \mathcal{M}\right\}$ as the set of feasible access control decision for the FBS. As mentioned before, the FBS's objective is to maximize the total utility of its FUs. Then, the optimal access control decision can be obtained by solving

$$
\begin{aligned}
\underset{\boldsymbol{a}_{F}}{\operatorname{maximize}} & \sum_{i \in \mathcal{F}} u_{i}\left(w_{i F}^{*}\left(x_{F}^{*}\left(\boldsymbol{a}_{F}\right)\right), P_{i}^{*}\left(w_{i F}^{*}\left(x_{F}^{*}\left(\boldsymbol{a}_{F}\right)\right)\right)\right) \\
\text { subject to } & \boldsymbol{a}_{F} \in \mathcal{A}_{F},
\end{aligned}
$$

where $P_{i}^{*}\left(w_{i F}^{*}\left(x_{F}^{*}\left(\boldsymbol{a}_{F}\right)\right)\right), w_{i F}^{*}\left(x_{F}^{*}\left(\boldsymbol{a}_{F}\right)\right)$ and $x_{F}^{*}\left(\boldsymbol{a}_{F}\right)$ are the optimal solutions to Stages IV, III and II, respectively, which can be obtained using equation (5), Algorithm 1, and equation (8), respectively. Note that the FBS prefers obtaining more resources from the MBS to serve its FUs. Then, the optimal solution to (9) can be found by searching among the association profiles in $\mathcal{A}_{F}$ and selecting the one that brings the largest amount of additional resources to the FBS. Specifically, for each association profile, i.e., $\boldsymbol{a}_{F}$, the number of channels allocated to the FBS is $x_{F}=\left\lceil\sum_{i \in \mathcal{F}} w_{i F}^{\min }\right\rceil+\left\lfloor\sum_{i \in \mathcal{S}_{F}^{m}} w_{i M}^{c}\right\rfloor$, and the additional resources the FBS obtains by accepting MUs can be represented as $\Delta \varphi\left(\boldsymbol{a}_{F}\right) \triangleq\left\lfloor\sum_{i \in \mathcal{M}} a_{i} w_{i M}^{c}\right\rfloor-\sum_{i \in \mathcal{M}} a_{i} w_{i F}^{\min }$, where the first term is the additional channels obtained from the MBS and the second term is the amount of resources to be allocated to the MUs (according to Section III-B). Therefore, the association profile that maximizes $\Delta \varphi\left(\boldsymbol{a}_{F}\right)$ is $\arg \max _{\boldsymbol{a}_{F} \in \mathcal{A}_{F}} \Delta \varphi\left(\boldsymbol{a}_{F}\right)$. Since we assume each FU can only access one channel at a time, it is not necessary for the FBS to obtain more than $|\mathcal{F}|$ channels to serve its FUs, where $|\cdot|$ is the cardinality of the set. We denote $\mathcal{A}_{F}^{\prime}$ as the set of association profiles which satisfies $\left\lceil\sum_{i \in \mathcal{F}} w_{i F}^{\min }\right\rceil+\Delta \varphi\left(\boldsymbol{a}_{F}\right)>|\mathcal{F}|$. It can be seen that if $\mathcal{A}_{F}^{\prime} \neq \emptyset$, accepting any association profile in $\mathcal{A}_{F}^{\prime}$ maximizes the total utility of FUs, since the resources available for the FUs are sufficient to guarantee that each FU obtains one channel. In this case, we choose the profile that contains the minimum number of MUs as the final decision. On the other hand, if $\mathcal{A}_{F}^{\prime}=\emptyset$, the optimal access control decision can be selected as the association profile that gives the largest $\Delta \varphi\left(\boldsymbol{a}_{F}\right)$. Therefore, the optimal access control decision can be represented as

$$
\boldsymbol{a}_{F}^{*}= \begin{cases}\arg \min _{\boldsymbol{a}_{F} \in \mathcal{A}^{\prime}} \sum_{i \in \mathcal{M}} a_{i}, & \text { if } \mathcal{A}^{\prime} \neq \emptyset \\ \arg \max _{\boldsymbol{a}_{F} \in \mathcal{A}_{F}} \Delta \varphi\left(\boldsymbol{a}_{F}\right), & \text { otherwise. }\end{cases}
$$

\section{E. Resource Management Mechanism}

We have derived the desired strategies for base stations and users in each resource management stage. The proposed resource management mechanism can be summarized as follows. i) Initialization: The MBS collects global information of the network, and calculates the resource allocation parameters $w_{i M}^{c}, \forall i \in \mathcal{M}$, as discussed in Section III-C. Then, the MBS sends the information to the FBS.

ii) Access Control Process: Each MU sends an access request to the FBS if it can obtain a larger utility. Then, the FBS determines its association decision according to (10), and sends the information of $\boldsymbol{a}_{F}$ to the MBS.

iii) Resource Allocation Process: The MBS determines the number of channel $x_{F}$ allocated to the FBS according (8). Then, the MBS and the FBS allocate their available resources to each associated user according to Algorithm 1.

iv) Power Control Process: Each user determines its optimal power according to (5).

Note that the proposed mechanism has a nice property, it encourages the FBS to use hybrid access control in order to improve the total utility of its FUs. On one hand, the FBS may obtain additional resources to serve its own FUs by accepting to serve nearby MUs, as discussed in Section III-D. On the other hand, an MU who communicates with the MBS with poor signal quality can improve its utility by accessing the nearby FBS without additional cost (i.e., extra payment).

\section{PERFormance Evaluation}

We evaluate the performance of the proposed resource management mechanism using simulation. We consider a system that consists of an MBS and an FBS, where the FBS is located at the center of a circular region whose radius is $30 \mathrm{~m}$. The distance between the MBS and the FBS is $160 \mathrm{~m}$. The radius of the femtocell is $10 \mathrm{~m}$, and 4 FUs are randomly distributed in it. The MBS serves 8 MUs who are randomly distributed in the circular region with the constraint that $\eta$ of them are indoor users who are located within the femtocell. There are 9 channels available for the system, each with a bandwidth of $180 \mathrm{kHz}$. The path loss exponent between a user and the MBS (or an FBS) is 4 (or 3), and we choose the wall penetration loss as $8 \mathrm{~dB}$. The users can choose transmission power from the set $\mathcal{P}=\{0,100 \mathrm{~mW}, 150 \mathrm{~mW}, 200 \mathrm{~mW}, 250$ $\mathrm{mW}$. The noise power is $-120 \mathrm{dBm}$. Each time slot is $1 \mathrm{~ms}$ and one transmission period consists of 100 time slots. We consider two types of applications, the MUs are requesting regular video chatting with a minimum rate requirement of 128 kbps. The FUs are requesting high definition video chatting 


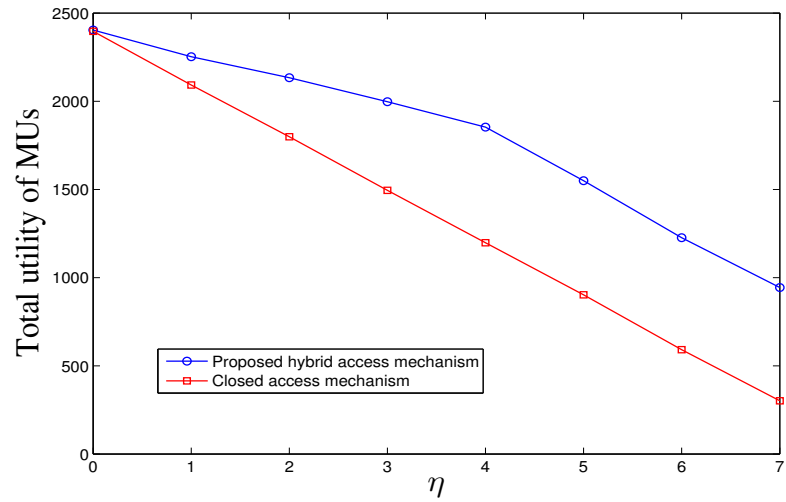

Fig. 3. Total utility of the MUs versus number of indoor MUs.

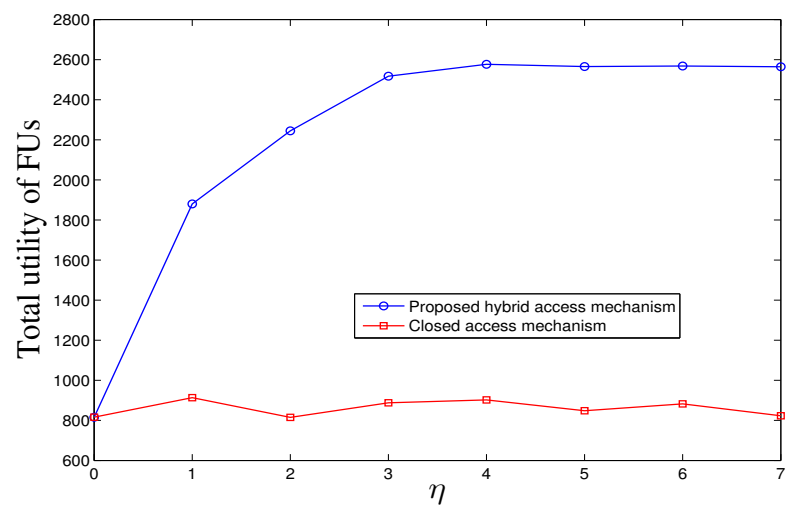

Fig. 4. Total utility of the FUs versus number of indoor MUs.

with a minimum rate requirement of $300 \mathrm{kbps}$. Other system parameters are $\alpha=200, \beta=0.1$ and $\gamma=100$. We evaluate the performance of the proposed configuration mechanism and the configuration mechanism with closed access with respect to different number of indoor MUs $(\eta)$, and the result is averaged over 50 simulation runs.

Fig. 3 shows that the total utility of MUs achieved by the proposed mechanism is higher than that achieved by the mechanism with closed access, and the performance gap between the two mechanisms first increases as the number of indoor MUs and then remains constant after $\eta=4$. This is because when using the mechanism with closed access, as the number of indoor MUs increases, the total utility of the MUs becomes smaller due to the poor signal quality from the MBS to the indoor MUs. However, with the proposed mechanism, the indoor MUs may switch to the femtocell to improve their utilities. Note that when $\eta>4$, it is not necessary for the FBS to accept more MUs, since the FBS already obtains sufficient channels to serve its FUs, as discussed in Section III-D. Therefore, the performance degradation for both mechanisms are the same when $\eta>4$.

Fig. 4 shows that as the number of indoor MUs increases, the total utility of FUs for the mechanism with closed access remains almost constant, since the FBS always obtains the minimum number of channels to serve its FUs. However, the total utility achieved by the proposed mechanism is increasing with $\eta$, which has significant performance improvement compared to the mechanism with closed access. The reason

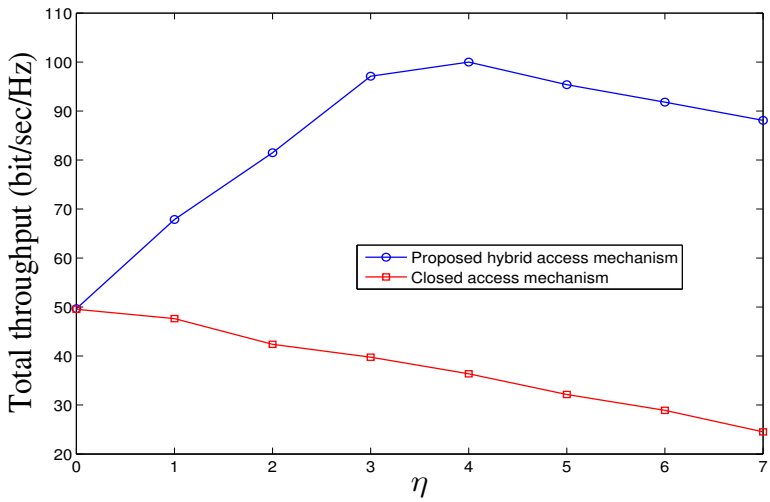

Fig. 5. System throughput versus number of indoor MUs.

is that when the FBS operates in the hybrid access mode, it can obtain more resources from the MBS by serving MUs. When $\eta>4$, the FBS has already obtains sufficient channels (4 channels) from the MBS to serve its FUs, and the total utility of FUs becomes almost a constant.

Fig. 5 shows the system throughput for both configuration mechanisms. On one hand, as the number of indoor MUs increases, the system throughput for the mechanism with closed access decreases. This is because more MUs experience poor signal quality from the MBS. On the other hand, the system throughput with proposed mechanism increases with the number of indoor MUs at the beginning and then decreases after $\eta$ reaching a threshold. The reason is as follows. With the proposed hybrid access mechanism, the FBS can obtain more channels to serve its own FUs by accepting to serve indoor MUs. Therefore, the FUs can obtain more resources as the number of indoor MUs increases, which result in higher data rates at the FUs. When the number of indoor MUs reaches 4 , the FBS does not accept more MUs, as explained for Figs. 3 and 4. Therefore, when $\eta$ is greater than 4, the additional indoor MUs can not be served by FBS anymore, and their data rates decreases due to the poor signal quality from the MBS, which also decreases the system throughput.

\section{CONCLUSION}

In this paper, we studied uplink resource management in a macro-femto system with hybrid access control. We proposed a four-stage resource management mechanism where base stations and users make decisions sequentially to optimize their own utilities. We found the optimal decisions at the base stations and users in each resource management stage. We showed that the proposed mechanism can provide incentives for the FBS to operate in hybrid access mode. Simulation results showed that the proposed resource management mechanism achieves higher utility at both the FBS and MBS than the mechanism with closed access, especially when the number of indoor MUs in the system is large. This work considered orthogonal channel allocation to avoid interference among MUs and FUs. It is also interesting to extend the mechanism with advanced interference management techniques, which will be considered in future work. 


\section{Appendix A: Proof of Theorem 1}

Proof: According to the discussion in Section III-B, we only need to prove that Algorithm 1 solves the transformed problem (7) optimally. We denote $\Delta \widetilde{u}_{i}(k)=\Delta u_{i}\left(\frac{k}{T}\right)$ as the utility increment when FU $i$ obtains its $k$ th time slot from the FBS. It can be shown that $\Delta \widetilde{u}_{i}(k)$ satisfies

$$
\Delta \widetilde{u}_{i}(k) \geq \Delta \widetilde{u}_{i}(k+1) \quad \text { if } w_{i F}^{\min } T<k<T .
$$

The proof of (11) is provided in Appendix B. We denote the set $\mathcal{T}=\{1,2, \ldots, T\}$, and define $W_{i}^{k}$ as an indicator such that $W_{i}^{k}=1$ indicates user $i$ obtains its $k$ th time slot $\left(W_{i}^{k}=0\right.$ otherwise). Then, problem (7) is equivalent to the following problem

$$
\begin{array}{cl}
\underset{W_{i}^{k}, i \in \mathcal{F}, k \in \mathcal{T}}{\operatorname{maximize}} & \sum_{i \in \mathcal{F}} \sum_{k \in \mathcal{T}} W_{i}^{k} \Delta \widetilde{u}_{i}(k) \\
\text { subject to } & \sum_{i \in \mathcal{F}} \sum_{k \in \mathcal{T}} W_{i}^{k} \leq T\left(x_{F}-\sum_{i \in \mathcal{S}_{F}^{m}} w_{i F}^{\min }\right), \\
& W_{i}^{k}=1, \quad \forall k \leq w_{i F}^{\min } T, i \in \mathcal{F}, \\
& \prod_{t=1}^{k} W_{i}^{t}=W_{i}^{k}, \quad \forall k \in \mathcal{K}, i \in \mathcal{F} .
\end{array}
$$

The first constraint indicates the total number of time slots allocated to the FUs is no larger than $T\left(x_{F}-\sum_{i \in \mathcal{S}_{F}^{m}} w_{i F}^{\min }\right)$. The second constraint indicates that user $i$ should obtain at least $w_{i F}^{\min } T$ time slots from the FBS to guarantee its QoS. The third constraint implies that if user $i$ obtains its $k$ th time slot from the FBS $\left(W_{i}^{k}=1\right)$, it should also obtain all the previous time slots $\left(W_{i}^{t}=1, \forall t \leq k\right)$. We define the set $\Delta \mathcal{U}=\left\{\Delta \widetilde{u}_{i}(k), \forall k \in\left\{w_{i F}^{\min } T+1, \ldots, T\right\}, \forall i \in \mathcal{F}\right\}$ and denote $\Delta \mathcal{U}^{\prime}$ as the set of the largest $T_{R}$ (defined in Algorithm 1) elements from $\Delta \mathcal{U}$. With property (11), it can be shown that if $\Delta \widetilde{u}_{i}(k) \in \Delta \mathcal{U}^{\prime}$, then $\Delta \widetilde{u}_{i}(t) \in \Delta \mathcal{U}^{\prime}, \forall t<k$. Therefore, the corresponding values $W_{i}^{k}=1$ associated with the positive elements $\Delta \widetilde{U}_{i}(k)$ in $\Delta \mathcal{U}^{\prime}$ constitute the solution to (12). Note that in Algorithm 1, for each remaining time slot after basic allocation, we allocate it to the user with the largest positive $\Delta u_{i}\left(w_{i F}+1 / T\right)$. Based on the non-increasing property of $\Delta u_{i}\left(w_{i F}\right)=\Delta \widetilde{u}_{i}\left(w_{i F} T\right)$, Algorithm 1 exactly finds the positive elements among the first largest $T_{R}$ elements in $\Delta \mathcal{U}$. Therefore, Algorithm 1 solves problem (12), which implies that it solves problem (7). This completes the proof.

\section{APPENDIX B: PROOF OF (11)}

Proof: Based on the definition of $\Delta \widetilde{u}_{i}(k)$, to prove (11) is equivalent to show that $u_{i}\left(w_{i F}, P_{i}^{*}\left(w_{i F}\right)\right)$ is a concave function of $w_{i F}$ in $\left[w_{i F}^{\min }, 1\right]$ (where we relax the constraint that $w_{i F}$ takes discrete values).

First, it can be shown that $P_{i}^{*}\left(w_{i F}\right)$ obtained in (5) does not increase with $w_{i F}$. We relax the integer constraint on $P_{i}$ and denote $\widetilde{P}_{i}=\arg \max _{P_{i} \in(0, \infty)} u_{i}\left(w_{i F}, P_{i}\right)$ given $w_{i F} \in$ $\left[w_{i F}^{\min }, 1\right]$. It can be verified that $\widetilde{P}_{i}$ satisfies $\left.\frac{d u_{i}}{d P_{i}}\right|_{P_{i}=\widetilde{P}_{i}}=0$, which leads to

$$
\frac{\alpha}{1+\widetilde{R}_{i F}-R_{i}^{\min }} \cdot \frac{B g_{i F}}{\ln 2\left(\sigma^{2}+g_{i F} \widetilde{P}_{i}\right)}-\beta=0
$$

If $\widetilde{P}_{i}$ increases with $w_{i F}$, equation (13) no longer holds, since the left side of the equation decreases as both $\widetilde{P}_{i}$ and $w_{i F}$ increase. Therefore, $\widetilde{P}_{i}$ is decreasing with $w_{i F}$. Note that $P_{i}^{*}\left(w_{i F}\right)$ is the special case of $\widetilde{P}_{i}$ when $\widetilde{P}_{i}$ takes values from $\mathcal{P}$. We can further conclude that as $w_{i F}$ increases from $w_{i F}^{\min }$ to $1, P_{i}^{*}\left(w_{i F}\right)$ either remains constant or decreases to the next power level in $\mathcal{P}$. We denote $P_{i}^{*}(1)=P_{l v}$ where $v \in$ $\{1,2, \ldots, L\}$. Then, for $w_{i F} \in\left[w_{i F}^{\min }, 1\right]$, the optimal power $P_{i}^{*}$ takes values in the set $\mathcal{P}^{\prime}=\left\{P_{l v}, P_{l(v+1)}, \ldots, P_{l L}, P_{\max }\right\}$. Each value $P_{i}^{*}$ in $\mathcal{P}^{\prime}$ is obtained when $w_{i F}$ falls within a continuous interval in $\left[w_{i F}^{\min }, 1\right]$, denoted as $\mathcal{W}\left(P_{i}^{*}\right)$. Thus, [ $\left.w_{i F}^{\min }, 1\right]$ is divided into $L-v+2$ non-overlapping intervals.

Next, for any interval $\mathcal{W}\left(P_{i}^{*}\right)\left(p_{i}^{*} \in \mathcal{P}^{\prime}\right)$ defined above, $P_{i}^{*}$ is a constant, we take second order derivative of $u_{i}\left(w_{i F}, P_{i}^{*}\right)$ respect to $w_{i F}$, which gives

$$
\frac{d^{2} u_{i}}{d w_{i F}^{2}}=-\left(\log _{2}\left(1+\frac{g_{i F} P_{i}^{*}}{\sigma^{2}}\right)\right)^{2} \frac{\alpha}{1+\widetilde{R}_{i F}-R_{i}^{\min }}<0 .
$$

Therefore, $u_{i}\left(w_{i F}, P_{i}^{*}\right)$ is a concave function of $w_{i F}$ in $\mathcal{W}\left(P_{i}^{*}\right)$. Note that $\bigcup_{P_{i}^{*} \in \mathcal{P}^{\prime}} \mathcal{W}\left(P_{i}^{*}\right)=\left[w_{i F}^{\min }, 1\right]$ and $u_{i}\left(w_{i F}, P_{i}^{*}\left(w_{i F}\right)\right)$ is a continuous function over $\left[w_{i F}^{\min }, 1\right]$ (after relaxing the discrete value constraint on $w_{i F}$ ). Based on (14), we conclude that $u_{i}\left(w_{i F}, P_{i}^{*}\left(w_{i F}\right)\right)$ is a concave function of $w_{i F}$ over $\left[w_{i F}^{\min }, 1\right]$. This completes the proof.

\section{REFERENCES}

[1] A. Barbieri, A. Damnjanovic, T. Ji, J. Montojo, Y. Wei, D. P. Malladi, O. Song, and G. Horn, "LTE femtocells: System design and performance analysis," IEEE J. Sel. Areas Commun., vol. 30, no. 3, pp. 586-594, Apr. 2012.

[2] V. Chandrasekhar, J. Andrews, and A. Gatherer, "Femtocell networks: A survey," IEEE Commun. Mag., vol. 46, no. 9, pp. 59-67, Sept. 2008

[3] J. G. Andrews, H. Claussen, M. Dohler, S. Rangan, and M. C. Reed, "Femtocells: Past, present, and future," IEEE J. Sel. Areas Commun., vol. 30, no. 3, pp. 497-508, Apr. 2012.

[4] N. Saquib, E. Hossain, L. B. Le, and D. I. Kim, "Interference management in OFDMA femtocell networks: Issues and approaches," IEEE Trans. on Wireless Commun., vol. 19, no. 3, pp. 86-95, June 2012.

[5] A. Golaup, M. Mustapha, and L. B. Patanapongpibul, "Femtocell access control strategy in UMTS and LTE," IEEE Commun. Mag., vol. 47, no. 9, pp. 117-123, Sept. 2009.

[6] G. de la Roche, A. Valcarce, D. Lopez-Perez, and J. Zhang, "Access control mechanisms for femtocells," IEEE Commun. Mag., vol. 48, no. 1, pp. 33-39, Jan. 2010.

[7] F. Pantisano, M. Bennis, W. Saad, and M. Debbah, "Spectrum leasing as an incentive towards uplink macrocell and femtocell cooperation," IEEE J. Sel. Areas Commun., vol. 30, no. 3, pp. 617-630, Apr. 2012.

[8] Y. Yi, J. Zhang, Q. Zhang, and T. Jiang, "Spectrum leasing to femto service provider with hybrid access," in Proc. of IEEE INFOCOM, Orlando, FL, Apr. 2012.

[9] S. Yun, Y. Yi, D.-H. Cho, and J. Mo, "Open or close: On the sharing of femtocells," in Proc. of IEEE INFOCOM, Shanghai, China, Apr. 2011.

[10] W. C. Cheung, T. Q. S. Quek, and M. Kountouris, "Throughput optimization, spectrum allocation, and access control in two-tier femtocell networks," IEEE J. Sel. Areas Commun., vol. 30, no. 3, pp. 561-574, Apr. 2012.

[11] P. Xia, V. Chandrasekhar, and J. G. Andrews, "Open vs. closed access femtocells in the uplink," IEEE Trans. on Wireless Commun., vol. 9, no. 12 , pp. 3798-3809, Dec. 2010.

[12] C. H. Ko and H. Y. Wei, "On-demand resource-sharing mechanism design in two-tier OFDMA femtocell networks," IEEE Trans. on Veh. Technol., vol. 60, no. 3, pp. 1059-1071, Mar. 2011.

[13] S. Shenker, "Fundamental design issues for the future internet," IEEE J. Select. Areas Commun., vol. 13, no. 7, pp. 1176-1188, Sept. 1995.

[14] D. Tse and P. Viswanath, Fundamentals of Wireless Communication. Cambridge University Press, 2005. 